\title{
Il futuro delle Società Scientifiche e la Società Italiana di Medicina di Laboratorio*
}

\author{
The future of scientific societies and the Italian Society for Laboratory Medicine
}

\author{
Piero Cappelletti
}

Ricevuto: 5 settembre 2011 / Accettato: 5 settembre 2011

(C) Springer 2011

Riassunto La creazione di una Società Scientifica è un momento essenziale nell'affermazione di una disciplina o di una specialità. Oggi le Società Scientifiche attraversano una crisi esistenziale legata alle mutate condizioni della medicina e della società, che mettono in discussioni i valori e i mezzi tradizionalmente utilizzati per rappresentare la disciplina o la specialità cui riferiscono. Le ragioni della crisi sono, dunque, pratiche e di sostanza e possono essere sintetizzate in: 1) comunicative, conseguenze dell'era elettronica (cyberconferenze che sostituiscono i meeting, giornali on line e open access, nuove forme di comunicazione come e-mail, blog e social network); 2) sociologiche, conseguenti ai mutamenti dell'organizzazione sanitaria (invecchiamento degli iscritti, crisi delle vocazioni, contrasto tra esigenze dei membri scientificamente attivi e di quelli non attivi); 3) culturali, che attengono ai valori disciplinari rappresentati e custoditi: la Società non aggiorna il corpus di conoscenze e competenze che connota la disciplina adeguandolo ai cambia-

\footnotetext{
* Rielaborato dalla relazione "Il ruolo delle Società Scientifiche nella formazione" tenuta al Convegno di Studio in memoria di Paolo Rizzotti LA SANITA' VERONESE E IL SISTEMA SANITARIO DELLA REGIONE VENETO - 11 dicembre 2009, Verona.
}

\section{P. Cappelletti}

IRRCCS CRO Aviano

Via Franco Gallini 2, 33081 Aviano (PN)

Tel.: +39-0434-659217

Fax: +39-0434-652182

e-mail: piero.cappelletti@cro.it menti della medicina e della società; la Società non è percepita come fonte della normatività professionale nei confronti del mercato sanitario; la Società non è percepita come un soggetto forte di advocacy. Nonostante ciò, un futuro esiste per le Società Scientifiche ed è cruciale per discipline e adepti. Le condizioni per garantirsi un futuro sono legate in primo luogo alla consapevolezza di rappresentare i valori core della disciplina, alla credibilità culturale e morale e indipendenza chiaramente percepibile da industria e fornitori, al mutuo scambio leadership-soci per la soluzione dei problemi organizzativi e professionali e alla trasparenza, democrazia e responsabilità della leadership. Sotto il profilo pratico, le Società Scientifiche devono approntare una advocacy in termini di diffusione della vision societaria e nuove forme di networking adeguate all'era elettronica (portale, giornali, meeting), inventare ruoli per gli "emeriti" e forme di stimolo per i "giovani" professionisti e un nuovo trade-off tra membri scientificamente attivi e non attivi, attuare una formazione "close to members" per bisogni, disegno formativo, verifica degli outcome e una assistenza ai soci per la ricerca, attenta a criteri metodologici (STARD), mentoring e codici di condotta.

Parole chiave Società Scientifiche · Advocacy · Formazione continua $\cdot$ Ricerca scientifica

Summary Since the Seventeenth Century, the birth of the scientific society has been a critical step in the success of a science, discipline, or speciality. Today, scientific societies are challenged by a membership crisis resulting from 
changes in medicine and society that have hit traditional values and the tools of the profession. The causes are multiple and are consequences of changes in communication in the digital era (cyber-conferences, open access journals, e-mail, blogs, social networks), sociological changes in the demography of members (ageing of members, new priorities of "trainees", different expectations of members working in science versus non-working members) and difficulties in interpretation of the core values of a scientific society. Other areas of difficulty include upgrading the body of knowledge and skills of the discipline, independence versus the non-independence in a commercialized health-care market, promotion and advocacy of the collective professional interest in relation to the public interest. Nevertheless, scientific societies are and will continue to be critical for disciplines and the experts in those disciplines. The conditions for the continuing relevance of a scientific society are awareness of the responsibility of representing the core values of the discipline, cultural and ethical reliability, a coordinated strategy for scientifically active and non-active members, and democracy and accountability in the governance of the society. From a practical point of view, scientific societies should promote and disseminate the vision of the discipline within medicine and health-care, apply new tools for networking (meetings and journals in the electronic era), develop new roles for ageing members, offer incentives to attract new subscriptions, ensure a balance between the interests of acti$v e$ and those of non-active members, ensure relevance in the medical education offered to members (personalized assessment of needs, redesign of programmes, outcome measurement), provide intelligent support for research in terms of methodological criteria, offer mentoring, and implement responsible codes of conduct.
Key words: Scientificy Society $\cdot$ Advocacy $\cdot$ Medical education $\cdot$ Scientific research

Fino dagli albori della medicina occidentale, tra il VII e il V secolo avanti Cristo, nelle isole greche e sulle coste dell' Asia Minore, i medici, così come i filosofi, si incontravano in cenacoli e in simposi per discutere delle teorie e della pratica della loro attività e per scambiarsi informazioni e suggerimenti sui casi difficili o particolari che avevano incontrato. Talora, questi gruppi di pari e di discepoli si trasformavano in rapporti continuativi dando vita ad "istituzioni" come la Scuola di Cnido e quella di Coo. Da sempre, dunque, filosofi, medici e scienziati hanno la necessità di stabilire legami di scambio culturale e professionale [1].

Le Società Scientifiche nell'accezione moderna sorgono con la rivoluzione scientifica del 1600 e in quel secolo, con 170 società operanti, l'Italia ne rappresentava il luogo di nascita e di massima espansione [2]. Nel secolo successivo l'Europa e il mondo anglosassone prendono progressivamente il sopravvento e nel XIX secolo, il momento dell'affermazione della medicina moderna e del sorgere delle specialità mediche, si avvia la diffusione delle società medico-scientifiche, che s'impenna nel XX secolo [3] (Fig. 1).

Oggi in Italia la Federazione Italiana delle Società Medico Scientifiche Italiane (FISM) [4], che peraltro non accoglie tutte le Società del settore, annovera 176 Società in rappresentanza di circa 120.000 soci. Tuttavia, negli ultimi decenni il numero della Società Scientifiche, e in particolare di quelle Medico-Scientifiche, tende a diminuire in tutto il mondo (Fig. 1), in rapporto ad una crisi che potremo definire "esistenziale", come ben illustrato dal seminario "The future of Learned and Professional Societies" organizzato nel 1999 dalla Foundation for

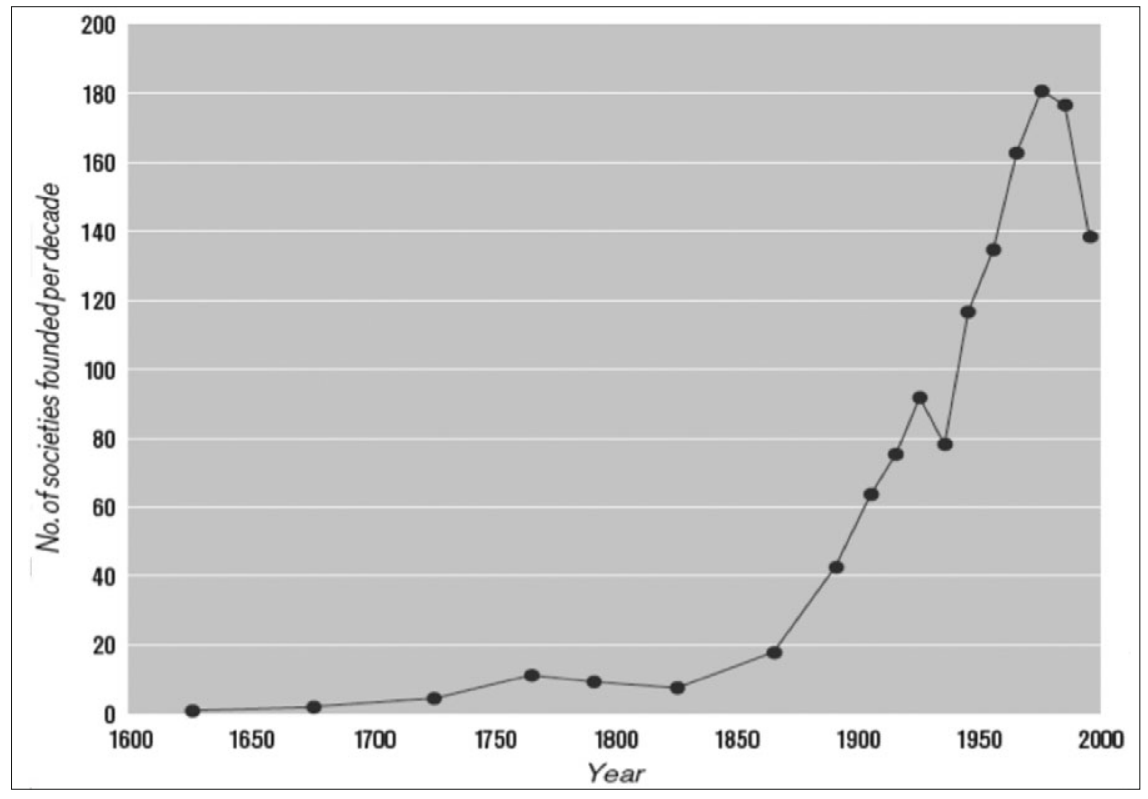

Fig. 1 Tasso di crescita di Società Scientifiche in epoca moderna e contemporanea. Tratta da http://www.lib.uwaterloo.ca/society [3] 
Science and Technology e dalla Association of Learned and Professional Society Publishers [5].

\section{Le ragioni della nascita delle Società Scientifiche (Tab. 1)}

È unanimemente accettato che la creazione di una Società sia un momento essenziale nell'affermazione di una disciplina o di una specialità. I componenti di una disciplina scientifica sono (o dovrebbero) essere legati l'un l'altro da simili aspirazioni, valori e cultura e costituire una comunità caratterizzata da un "unum sentire", rispetto ad obiettivi condivisi, al loro valore e ai mezzi appropriati per raggiungerli e rispetto al genere di relazioni da stabilire tra i componenti l'associazione e, spesso, tra essi e gli "altri". Le discipline scientifiche sono un gruppo di riferimento fortemente normativo, i cui valori e standard servono da guida per i singoli scienziati nell'organizzare e condurre il proprio lavoro e, per gli esterni al gruppo, per comprenderne e valutare le loro attività. La Società Scientifica, quale istituzione visibile, stabile e durevole, agisce come custode dei valori core della disciplina, delle sue tradizioni distintive e del corpo unitario di conoscenza e abilità che la connota. Essa funziona come una essenziale sorgente di identità per il singolo aderente, consentendogli di mantenere la consapevolezza di appartenere ad una ricca e rispettata tradizione, così come la certezza della sua continua evoluzione. La sintesi dei valori professionali e delle norme di comportamento prodotta dai membri della Società Scientifica e trasmessa ai singoli componenti è un mezzo efficace per diffondere e condividere il sentimento comune, così che, anche se la disciplina e la Società non producono le nuove generazioni di scienziati per via biologica, lo fanno per via sociale. Inoltre, il "gruppo" garantisce che il singolo si comporti, almeno sotto il profilo scientifico e professionale, secondo le regole e le obbligazioni definite nel modo predetto dalla disciplina. Le Società Scientifiche sono, dunque, guardiani, la cui sorveglianza delle relazioni tra i membri e tra gli appartenenti e gli "altri" è critica nel governare il progresso della scienza [6].

Sintetizzando al massimo le ragioni per le quali viene fondata una Società Scientifica, possiamo indicare da un lato l'advocacy, intesa come difesa degli interessi professionali e forum di standard e di arbitrato, e dall'altro il networking, come scambio di informazioni e ricerche, conoscenze e competenze utili sul piano professionale e scientifico [7]. Sottolineano Pellegrino e Relman [8] che la storia delle associazioni professionali mediche riflette una tensione costante tra l'interesse individuale e disciplinare e le idee etiche, conflitto che non ha mai avuto definitiva composizione.

La maggior parte delle Società Scientifiche e Medico Scientifiche è nata per favorire lo scambio informativo e formativo tra i soci (networking) negli incontri di varia natura (convegni, simposi, congressi da un lato, gruppi di lavoro e di studio dall'altro) e con la offerta di mezzi di comunicazione permanente, tipicamente i giornali scientifici e, oggi, i mezzi offerti dalla rivoluzione di internet e della rete [9]. I meeting sostenuti dalle Società Scientifiche sono (o dovrebbero essere) più aderenti agli obiettivi della disciplina e alle necessità degli adepti e indipendenti dalla pressione commerciale dell'Industria. La partecipazione ai meeting è, per universale considerazione, un'esperienza essenziale nello sviluppo professionale, sia perché rappresenta la più tradizionale e solida maniera di presentare e discutere i risultati scientifici dei diversi adepti sia perché è la sede ideale dell'interscambio e del "riconoscimento" professionale [10]. Da questo punto di vista, i rapporti informali e il confronto individuale sulla pratica professionale sono almeno altrettanto importanti della trasmissione formale dei risultati scientifici nelle sessioni congressuali, delle "evidenze" e delle linee guida costruite o approvate dalla Società. La collegialità professionale è uno strumento cruciale dello sviluppo della scienza e della professione, perché gli umani sono animali sociali, come già diceva Aristotele, e il progresso scientifico si attua "standing on the shoulders of giants", secondo la descrizione di Newton. Se l'interazione è interpersonale, il processo di interscambio e di crescita culturale è anche più gratificante. Inoltre, la partecipazione a comitati, pannel o gruppi di studio e di lavoro è una forma di "riconoscimento" che coinvolge i componenti non solo rispetto al tema specifico ma, in generale, nella convinzione di condividere una comune responsabilità nel "costruire" la scienza e la pratica professionale [10].

Il tradizionale strumento disciplinare e societario di condividere le esperienze scientifiche è rappresentato dai

Tabella 1 Ragioni per la nascita e la partecipazione a una Società Scientifica

Rappresentazione della disciplina o specialità

Scambio informativo e formativo (networking)

Difesa degli interessi disciplinari e professionali (advocacy)

Ruolo nella ricerca scientifica e nella sua applicazione

Ruolo nella formazione e nella definizione di qualità e competenza

Ruolo sociale
Organizzazione societaria

Meeting, gruppi di studio, giornali

Arbitrato, lobbying, standard professionali

Codici di comportamento, mentoring, EBM, linee guida

Training \& education

Educazione dell'opinione pubblica 
giornali e per molti secoli questo è stato il principale mezzo di diffusione scientifica, di disseminazione culturale e di democratizzazione della scienza. I giornali delle Società Scientifiche sono lo strumento per delineare le strategie scientifiche della disciplina, impostare cioè il dibattito scientifico e indirizzare la ricerca sulla base delle necessità concettuali piuttosto che su quelle mercantili tipiche dei giornali prodotti da Editori commerciali, per sottolineare i problemi "reali" della pratica professionale, per definire nuove aree di ricerca e per suggerire soluzioni basate sulle "evidenze" e sulle linee guida condivise [10]. Soprattutto nel passato, per molte Società Scientifiche la pubblicazione di riviste è stata anche una delle principali fonti di sostentamento economico [9]. Più recentemente le Società Scientifiche hanno dovuto far riferimento ad Editori professionali e ciò ha indotto molte tensioni nel mondo della editoria medico-scientifica, rispetto alle quali sono state suggerite linee di comportamento specifiche atte a salvaguardare da un lato la redditività della pubblicazione $\mathrm{e}$, dall'altro, l'indipendenza dei contenuti scientifici [11]. Benchè questa tensione dinamica possa produrre risultati positivi in termini di progresso scientifico, le condizioni alle quali ciò può avvenire appartengono al mondo delle buone intenzioni (are our words...ultimately self-serving or do they benefit the patient we profess to serve?) [11] piuttosto che a quello della realtà. Inoltre, lo stato dell'editoria medicoscientifica oggi pone con evidenza i problemi sollevati da Pellegrino e Relman [8] nel 1999: idealmente un giornale dovrebbe sopravvivere grazie agli abbonamenti o al sostegno della Società; la pubblicità e i sostegni dell'Industria dovrebbero essere limitati; i contenuti scientifici dovrebbero essere validati da una peer review o da una valutazione societaria, a seconda della tipologia e dell'impatto del lavoro in esame. Ma oggi le problematiche editoriali sono anche altre e conseguenti alla più grande rivoluzione nel campo dopo Gutenberg: la migrazione della parola dalla carta alla rete, l'accessibilità continua di gran parte delle pubblicazioni, la possibilità di scambio diretto con gli Autori e i "pari" attraverso $e$ mail, blog e social network [12].

Le Società Scientifiche sono uno strumento importante di difesa della disciplina e dei suoi adepti. Per advocacy si intende il processo politico con cui un singolo o un gruppo tendono ad influenzare la politica e l'allocazione di risorse all'interno dei sistemi e delle istituzioni politiche, economiche e sociali e che è motivato da principi etici e morali o semplicemente dalla protezione di interessi. Fare lobby è una forma semplice di advocacy. È evidente, peraltro, che la difesa anche economica delle prerogative della disciplina e dei suoi adepti non è di tipo rivendicazionistico e non attiene alla materia più tipicamente sindacale [8]. Le Società Scientifiche non devono legarsi al sindacato o cercare di diventare dei sindacati in quanto devono mantenere l'attenzione di tutti gli associati al miglioramento della qualità (per le Società Mediche della qualità della cura del paziente) e al mutuo supporto tra colleghi. Nella sua accezione più alta, l'advocacy rappresenta il tentativo di cambiare "ciò che è" in "ciò che dovrebbe essere", nella convinzione che il "dover essere" sia più giusto per tutta la società. Gli "advocacy groups" non vogliono governare ma vogliono influenzare la politica di governo e costituiscono un elemento essenziale della democrazia odierna [13].

La difesa esterna degli interessi della disciplina va di pari passo con la definizione delle regole e degli standard professionali e scientifici. La New Jersey Medical Society, prima associazione medica statunitense del 1766, nacque intorno ai temi più scottanti della professione: norme per la pratica clinica, standard educazionali, regole tariffarie e codice etico. Anche se alcuni di questi obiettivi sono oggi anche in Italia tipici degli Ordini, la questione della condotta degli adepti resta centrale per le Società Scientifiche per quanto riguarda le caratteristiche sia scientifiche che professionali dei componenti della disciplina di riferimento. In rapporto agli aspetti scientifici e di ricerca, le Società Scientifiche hanno un ruolo evidente nella definizione dei criteri e dei codici etici della ricerca e nella diffusione delle pratiche cliniche validate [8].

Le Società Scientifiche dovrebbero, sul piano della ricerca, impegnarsi a definire e articolare standard prodotti dal consenso interno alla disciplina e testimonianze del riconoscimento collettivo delle responsabilità etiche, incoraggiare il mentoring di abilità e pratica tra iscritti con diverso grado di preparazione ed esperienza, promuovere l'integrità della ricerca e sostenere lo svelamento di comportamenti scientificamente scorretti [6]. Sotto questo profilo molto è cambiato, almeno di là dell'oceano, negli ultimi decenni [14]. Se una ricognizione della AAAS (American Association for the Advancement of Science) nel 1980 rivelava che le Società Scientifiche dedicavano all'etica professionale "little attention and only minimal resources", una seconda ricognizione condotta tra il 1999 e il 2000 ha mostrato che il $68 \%$ delle Società intervistate aveva adottato un codice etico. Il ruolo delle Società Scientifiche nella definizione e diffusione di regole per la ricerca (responsible conduct of research, RCR) può esplicarsi lungo tre direttrici: la creazione e diffusione di codici etici, la pubblicazione di linee guida relative alla ricerca scientifica (norme per gli autori, responsabilità nel mentoring, ecc.), lo sviluppo e la disseminazione di materiali educativi. In particolare le Società Scientifiche dovrebbero sensibilizzare i propri aderenti riguardo ad alcuni indicatori di ricerca scientifica scorretta (Tab. 2) [15]. Gli Autori che si sono dedicati 
Tabella 2 Indicatori di correttezza nella ricerca. Modificata da Levine FJ, Iutcovich JM (2003) [15]

Autenticità del lavoro

Esperienza adeguata

Ruolo effettivo nella ricerca

Accesso e condivisione dei dati

Protezione degli umani e/o animali

Privacy e confidenzialità
Fabbricazione dei dati

Falsificazione dei dati

Plagiarismo

Appropriazione di dati altrui

Accuratezza nel reporting

Duplicazione delle pubblicazioni a questi aspetti sottolineano la complessità di impostare e valutare 1'azione delle Società Scientifiche in questo campo. Suggeriscono, inoltre, la costituzione di Comitati Etici Societari, composti anche da esperti non componenti della Società, sia per i progetti di ricerca scientifica sia per la definizione e valutazione di trial clinici, e della necessità dell'introduzione di nuove procedure, tecnologie e farmaci [16].

Sul piano della applicazione clinica, le Società Medico Scientifiche in particolare hanno l'obbligo etico di sorvegliare la qualità dei risultati di ricerca pubblicati, anche semplicemente applicando il rispetto dei criteri di buona ricerca come lo STARD, di contribuire alla definizione delle "evidenze" secondo i criteri della Evidence-Based Medicine (EBM), di difendere i fondamenti della EBM da ricorrenti attacchi post-moderni, di definire o contribuire a definire o approvare e diffondere linee guida (GL) fondate sulle evidenze, di monitorarne l'applicazione tra i soci e nella disciplina tramite indagini a questionario rivolte alla intera platea degli adepti o tramite valutazioni a distanza di tempo di campioni rappresentativi [17].

Queste attività saldano gli aspetti della ricerca a quelli della pratica clinica, degli standard professionali e dei modi in cui i professionisti acquistano e mantengono le loro conoscenze e competenze. Si tratta dell'altra faccia dell'attività scientifica delle Società Scientifiche: la formazione.

Nonostante alcune opinioni diverse da parte dell'Accademia, è pienamente accettato che le Società Medico Scientifiche abbiano un ruolo predominante nella formazione post-istituzionale e nel mantenimento e miglioramento di conoscenze e competenze lungo tutta la vita del professionista, secondo i dettami della continuous medical education (CME), lifelong learning for updating knowledge, e del continuous professional development (CPD), broader skills including management, education and training, information technology, audit, communication, team building [18].

È ben vero che il CPD, complesso di mezzi educativi con cui il professionista sviluppa, aggiorna e potenzia il modo con cui applica conoscenze, abilità e attitudini, cioè competenze, nella propria vita lavorativa, è un programma personale [19]. Ma la Società Scientifica è l'ambiente più adeguato per il professionista dove trovare $i$ mezzi per il suo CPD, proprio per le caratteristiche di identità disciplinare, comune sentire, obiettivi etici e assenza di interessi commerciali che ne fanno un'isola rispetto al mercato della salute che caratterizza l'epoca in cui viviamo. Gli strumenti di CME applicabili sono fondamentalmente i congressi/convegni, i corsi residenziali e la formazione a distanza (FAD) e in Italia sono normati dalle regole, recentemente rinnovate, della ECM nazionale e regionale.

In questo quadro il ruolo delle Società Scientifiche può essere così sintetizzato: 1) proporre contenuti e strategie formative all'ECM nazionale, monitorandone l'applicazione e i limiti; 2) coordinare le attività CME a livello europeo, mirando ad una omogeneità e interscambio formativo, insieme con altri attori (Ordini, e così via) [19]; 3) gestire attività formative (ECM e non) per i propri soci e per i non soci, individuando le necessità formative collettive, e offrendo percorsi flessibili e individualizzabili ai singoli soci; 4) contribuire a valutare l'efficacia delle strategie formative in generale e nei singoli gruppi, sperimentando la valutazione a distanza della formazione ai diversi livelli della conoscenza, abilità, competenze ed esiti.

Vi è, infine, un'ultima motivazione per la nascità delle Società Scientifiche, che alcuni Autori [10] identificano come separata da quelle finora elencate, ma che, a mio avviso, va inquadrata negli aspetti più alti della advocacy: il ruolo sociale delle Società Scientifiche che si sostanzia nell'educazione scientifica della pubblica opinione. Si è notato da più parti $[11,20]$ quanto sia ironico che proprio nel secolo in cui la scienza e la medicina consentono un miglioramento mai visto prima delle condizioni di vita e di benessere (almeno nel cosidetto "mondo occidentale"), sia sorta e alligni una "antiscienza" che si nutre di pregiudizi, pettegolezzi e fanatismo piuttosto che di logica razionale. Non sono mancate corresponsabilità nel mondo medico, sia nell'alimentare ragionamenti di dubbia efficacia sia nel celebrare come 
trionfali vittorie limitati avanzamenti terapeutici [20]. 亡̀ dunque essenziale che le discipline e le Società Scientifiche offrano "evidenze" di contenuto e di metodo al dibattito pubblico e politico sulla scienza, la tecnologia e la sanità $[10,11,20]$.

\section{La crisi delle Società Scientifiche (Tab. 3)}

La più interessante ricerca sul ruolo delle Società Medico Scientifiche e sulle motivazioni dell'associazione ad esse apparsa in letteratura negli ultimi anni è l'indagine [21] condotta dalla Società Tedesca di Dermatologia (DDG). Il questionario distribuito a 3.277 dermatologi tedeschi nel settembre del 2008 consisteva di 9 domande per saggiare i motivi di iscrizione, 13 per valutare l'immagine della Società presso gli iscritti, 7 per pesare l'utilizzo dei servizi offerti e 10 per chiarire le attese dei membri. La valutazione delle risposte avveniva attraverso una scala Likert a 4 punti. Inoltre il questionario dava la possibilità di risposta aperta a due domande riguardanti aspettative e futuro. I membri, di cui si raccoglievano alcuni dati anagrafici, erano divisi in due gruppi, definiti dalla risposta alla domanda riguardante l'attività scientifica del singolo: il gruppo W (scientificamente attivi) e il gruppo non-W (non scientificamente attivi). Il tasso di risposta è stato del $28,4 \%$ (931 iscritti) con il 46,7\% nel gruppo W e il 51,8\% nel gruppo non-W. Il campione era rappresentato prevalentemente da maschi $(57,1 \%)$, di età tra i 40 e i 49 anni $(43,3 \%)$, dedicati alla attività ambulatoriale $(59,6 \%)$ piuttosto che a quella ospedaliera $(31,3 \%)$ e per la gran parte iscritti da tempo alla Società $(83,7 \%>5$ anni).

Per quanto riguarda i motivi di iscrizione alla DDG, le ragioni più votate dai rispondenti sono state "la possibilità di educazione continua" e la "informazione continua sugli sviluppi della disciplina", mentre le meno condivise sono state "la possibilità di indirizzare politicamente la specialità", "l'utilità nella carriera professionale", "l'iscrizione in quanto raccomandata". Per quanto riguarda l'immagine che i soci hanno della Società, gli item più condivisi sono stati il "commitment" verso stan- dard elevati medici e disciplinari e, con minor forza, "l'impegno alla difesa della specialità", "lo sviluppo internazionale", "piattaforma valida per il dialogo disciplinare", "l'offerta di CME", “ la promozione della scienza e della ricerca". Per quanto riguarda l'utilizzo dei servizi offerti, il giornale è stato la risposta prevalente, mentre le risposte meno condivise sono state quelle relative ai presunti vantaggi alla partecipazione in pannel o gruppi di lavoro e all'utilizzo dei servizi degli uffici della DDG. Infine, per quanto riguarda le aspettative, le indicazioni più condivise sono state quelle di una maggior attenzione ai problemi di politica sanitaria, di costruzione e implementazione di linee guida e di formazione, intesa come training e qualificazione. I dati derivanti dalle domande aperte hanno confermato l'interesse prevalente per la politica sanitaria e professionale e per la formazione continua, mentre l'interesse per l'internazionalizzazione è apparso del tutto minoritario.

È molto interessante notare come le risposte nei due gruppi $\mathrm{W}$ e non-W siano fortemente diverse. Il gruppo non-W ha sottolineato particolarmente, a differenza del gruppo W, la possibilità di CME. Mentre il gruppo W ha valutato positivamente i seguenti item che il gruppo non$\mathrm{W}$ ha invece valutato come poco importanti: l'indirizzo politico della specialità, il supporto scientifico, lo scambio con i colleghi, l'utilità per la carriera professionale, l'informazione sugli sviluppi disciplinari, i vantaggi della partecipazione a pannel o gruppi di lavoro, la promozione del networking scientifico, il supporto e la promozione della ricerca, la qualificazione a posizioni di leadership, l'internazionalizzazione.

Dai dati complessivi di questa approfondita e articolata indagine, che non si riescono facilmente a condensare, appaiono confermate alcune considerazioni che sono state fin qui espresse sul ruolo delle Società Scientifiche e sui motivi di associazione e di crisi che incontriamo anche in Italia nella vita di tutti i giorni delle Società Scientifiche. In particolare, però, la diversità delle opinioni espresse dai membri scientificamente attivi (gruppo W) e quelli scientificamente non attivi (gruppo non-W) sottolinea che le Società Medico Scientifiche sono costantemente un' area di conflitto di interessi diversi, per

Tabella 3 Le ragioni della crisi delle Società Medico Scientifiche

\begin{tabular}{ll}
\hline Sociologiche & Invecchiamento degli iscritti \\
& Crisi di vocazioni \\
& Contrasto tra esigenze dei membri scientificamente attivi e di quelli non attivi \\
Comunicative & Teleconferenze vs meeting \\
& Giornali on line e open access \\
& Nuove forme di interazione (e-mail, blog, social network) \\
& La Società non aggiorna il corpus di conoscenze e competenze che connota la disciplina, adeguandolo ai cambiamenti \\
della medicina e della società & La Società non è percepita come fonte della normatività professionale vs il mercato sanitario \\
& La Società non è percepita come un soggetto forte di advocacy
\end{tabular}


i quali è necessario trovare un trade-off continuamente riequilibrato. I componenti della Società meno interessati agli aspetti scientifici sono anche meno interessati alla partecipazione alle sue articolazioni ma più interessati all'utilizzo dei suoi servizi, principalmente in termini di formazione accreditata (giornale, CME), di difesa degli aspetti professionali e di intervento nella politica sanitaria. I componenti scientificamente attivi sono invece fortemente attratti dal networking scientifico e dalle sedi più classicamente usate, quali i gruppi di lavoro, e dai riflessi scientifici, culturali e accademici di questi scambi e quindi sono più interessati alla Società come fornitrice infrastrutturale di opportunità in questo campo e come promoter di ricerca e di integrità della stessa, nel campo nazionale e internazionale.

Benchè la valutazione della DDG da parte dei suoi iscritti sia positiva, le risposte indicano che il futuro di essa è fortemente influenzato dalla capacità di dare risposte differenziate a componenti con background professionali e personali fortemente differenziati. Le linee d'azione dovranno quindi essere multiple e rivolte alla promozione della comunicazione professionale, all'apertura di dialogo per i gruppi di lavoro, alla revisione delle strategie formative, alla qualificazioni di docenti, alla definizione dei rapporti con l'Industria, all'internazionalizzazione. Le linee di intervento sono, come appare, anche parzialmente contraddittorie e l'equilibrio del mix sarà essenziale per evitare crisi di vocazione.

Nelle ultime cinque decadi il tasso di crescita delle Società Scientifiche si è negativizzato: nuove Società si formano per dare riferimento a nuove branche della scienza o a nuovi gruppi di interesse, ma molte delle Società presenti nel passato falliscono nel sostenere $\mathrm{i}$ cambiamenti del nuovo millennio [9]. Alcune ragioni sono meramente pratiche e, in buona sostanza, economiche e sono relative alle difficoltà di gestire adeguatamente il networking, attraverso l'offerta di meeting di contenuto ma anche attraenti e il mantenimento di riviste di qualità ma accessibili, di fronte al cambiamento mediatico indotto dalla teleconferenze, da internet e dalle nuove modalità di comunicazione, sempre accessibili e spesso gratuite [9]. Ma, anche se l'era elettronica sta cambiando radicalmente il ruolo delle Società Scientifiche nella disseminazione della scienza e nel networking degli adepti, le ragioni più profonde di associazione (advocacy, nella sua interpretazione di difesa di interessi ma anche di sviluppo in termini professionali e sociali) sono messe in crisi, in particolare, per i giovani professionisti, dalla difficoltà delle Società di spiegare il ruolo cruciale della partecipazione societaria per la crescita scientifica e professionale dei singoli. L'associazione ad una Società Scientifica, infatti, ha due facce: da un lato l'opportunità offerta, in termini di occasioni di scambio a prezzi conte- nuti, e, dall'altro, la responsabilità di agire collettivamente nella definizione degli standard scientifici e professionali a cui ispirarsi e da affermare come irrinunciabili per la società e i pazienti [10]. Ma ciò non viene trasmesso dalle Società, per un difetto di comunicazione e anche di consapevolezza del ruolo di guardiani della disciplina.

La crisi delle Società Scientifiche appare, dunque, da un lato, risiedere nell'inaridirsi delle tradizionali tipologie di incontro e di comunicazione, indotto dalla rivoluzione mediatica, e dalle ragioni di associazione legate al networking, a cui sono particolarmente sensibili i giovani professionisti. Infatti, nella ricerca effettuata da Bahr [22] su 45 trainees (graduate e post-doctoral students), la partecipazione al Congresso Annuale della SSR (Society for the Study of Reproduction) è apparsa essere la motivazione principale per l'iscrizione alla Società, nonché la seconda risposta alle domande "cosa può fare la Società per favorire la cariera dello specializzando/specializzato" e "cosa può fare il trainee per migliorare l'attrattività e la rilevanza dela SSR", mentre il networking è apparso essere la seconda ragione per l'iscrizione e la prima risposta alle domande "qual'è il valore derivante dall' associarsi a SSR" e "cosa può fare la Società per favorire la carriera dello specializzando/specializzato". Le difficoltà di ammodernare gli strumenti societari, soprattutto per renderli attrattivi per i giovani, si inseriscono nel quadro più generale del mutamento sociologico che tocca (anche) le Società Medico Scientifiche: invecchiamento degli iscritti, carenza di vocazioni, difficoltà nel combinare le esigenze dei soci scientificamente "attivi" e di quelli "passivi" [21].

D'altro canto, la crisi appare risiedere nell'incapacità delle Società di far percepire l'importanza di partecipare ad una istituzione che rappresenti unitariamente il sapere della disciplina, ne mantenga alti i valori pur adattandoli all'evoluzione della medicina e della società e sia sede della normatività scientifica della disciplina in quanto svincolata dagli interessi tipici del "mercato sanitario" [23]. Questa crisi di credibilità della Società Medico Scientifiche ha a che fare con aspetti che rappresentano due facce della stessa medaglia: la difficoltà di rappresentare in modo solido ma evolutivo "cosa è" la disciplina e la difficoltà di presentarsi come il riferimento indipendente di normatività professionale.

La professione medica, così come le sue declinazioni disciplinari, è da secoli definita dal fatto che i suoi adepti professano l'aderenza a standard di condotta nella pratica, che l'organizzazione rappresentativa della professione ha compiti riconosciuti deontologici (per la professione medica vi è l'Ordine; per la ricerca e la formazione delle discipline dovrebbero essere le Società Scientifiche) e che esiste un corpo di conoscenze e com- 
petenze che la connota e che viene continuamente aggiornato e condiviso dal lavoro dei propri aderenti [11]. D'altra parte, le definizione dei contenuti, confini e prospettive della disciplina per essere credibile deve essere trasparente e provare che avviene sulla base di un'indagine corretta della specialità e dei bisogni degli specialisti e non perché influenzata dall'esterno [23].

Ciò ha posto recentemente con forza il tema del rapporto delle professioni e delle Società Medico Scientifiche con l'Industria. Si riconosce universalmente il ruolo dell'Industria nel promuovere il progresso medico e l'opportunità di collaborazione tra Industria e Società Scientifiche, ma il livello a cui stabilire come distinguere correttamente formazione e marketing, quali possano essere gli effetti di regali, grant e consulenze, come salvaguardare linee guida da influenze dirette o indirette (trials e ricerche commissionate) del commercio è materia bollente. Se da un lato è ormai ampiamente diffusa (anche in Italia?) la pratica della dichiarazione del conflitto di interessi (per le pubblicazioni impostato secondo le linee ICMJE [24]), dall'altro si sono andate suggerendo pratiche più radicali per evitare totalmente il conflitto di interessi. Nel 2009 lo IOM (Institute of Medicine) ha pubblicato uno statement "Conflict of interest in medical research, education, and practice" [25] che suggerisce di sviluppare CME del tutto priva di supporto dell'Industria, pur riconoscendo che ciò comporterà più elevati costi per i professionisti e tagli nei costi per i provider di formazione, e di costruire linee guida da parte delle Società Scientifiche senza accettare fondi dall'Industria e senza coinvolgere nei pannel professionisti con conflitto di interesse. Sempre nel 2009 JAMA ha pubblicato una proposta molto stringente per il controllo del conflitto di interessi delle PMA (Professional Medical Associations) da parte di Rothman et al. [26]. Il supporto dell'Industria alle Società sul badget generale dovrebbe essere zero, eccetto gli introiti della pubblicità sui giornali e la vendita di spazi per la mostra dei prodotti in occasioni dei congressi. Nei meeting nazionali e regionali non dovrebbero esservi influenze industriali (anche come sponsorizzazioni) nei programmi e sui relatori/moderatori (scelta, supporto economico, regali), nep- pure nei simposi satelliti. Le PMA non dovrebbero accettare fondi per ricerche specifiche. L'Industria non deve sostenere iscrizioni e programmi formativi. Le PMA non devono supportare prodotti industriali. Per la costruzione di linee guida la dichiarazione di conflitto di interessi non è abbastanza, ci vuole l'assenza del conflitto. Nelle pubblicazioni la pubblicità deve essere chiaramente identificabile. Tutto quanto è proibito alle PMA non deve avvenire neanche tramite Società o Fondazioni affiliate. Gli organi delle PMA (Presidente e Board) non devono avere conflitto di interesse personale durante il loro mandato e, idealmente, per i due anni precedenti alla loro elezione. Infine, ogni PMA deve definire e diffondere una guida sul tema per i propri iscritti.

Pur comprendendo la vischiosità di alcune relazioni tra il mondo dell'Industria e quello delle Società Scientifiche, si dovrebbero avere chiare anche le conseguenze specifiche e generali di tali proposte radicali. Nel campo della formazione, ad esempio, ciò determinerebbe la chiusura dei professionisti all'interno delle singole strutture di appartenenza con un effetto di provincializzazione e autoreferenzialità, che in tutti questi anni si è cercato faticosamente di superare. Per le Società Scientifiche sarebbe, probabilmente, un ritorno alle origini, quando gli associati alla Plymouth Medical Society si ritrovavano il venerdì sera più prossimo alla luna piena per garantirsi che il chiaro di luna illuminasse la loro strada.

Tuttavia è evidente che solo la trasparenza e l'indipendenza garantiscono la credibilità. Le Società Scientifiche devono definire la propria agenda, le proprie priorità e le proprie strategie nel campo professionale, della formazione, della ricerca e delle prospettive societarie, dimostrando che l'Industria non le altera, direttamente o indirettamente, tramite sponsorizzazioni e supporti finanziari [27].

\section{Il futuro possibile delle Società Medico Scientifiche} (Tab. 4)

Come si è visto, le ragioni della crisi delle Società

Tabella 4 Linee per un futuro delle Società Scientifiche

Consapevolezza di rappresentare i valori core della disciplina

Credibilità culturale e morale e indipendenza da industria e fornitori chiaramente percepibile

Mutuo scambio leadership-soci per la soluzione dei problemi organizzativi e professionali

Trasparenza, democrazia e responsabilità della leadership

Advocacy in termini di diffusione della vision societaria

Nuove forme di networking adeguate all'era elettronica (portale, giornali, meeting)

Inventare ruoli per gli "emeriti" e forme di stimolo per i giovani professionisti

Nuovo trade-off tra membri scientificamente attivi e non attivi

Formazione "close to members" per bisogni, disegno formativo, verifica degli outcome

Ricerca attenta a criteri metodologici (STARD), mentoring e codici di condotta 
Medico Scientifiche sono molto simili in tutto il mondo occidentale e nelle diverse aree della Medicina e riflettono cambiamenti comunicativi rivoluzionari e modificazioni della demografia degli operatori sanitari, legate alle trasformazioni sociali in corso, nonché alle difficoltà culturali delle Società ad interpretare correttamente il loro ruolo alto di rappresentanti e guardiani dei contenuti scientifici, professionali ed etici della disciplina o specialità cui riferiscono.

Ciò impone alle Società Scientifiche di programmare strategie multiple d'azione, data la multifattorialità della crisi, e di affrontare il tema del senso più profondo della loro esistenza.

Sotto il profilo sociologico, non vi sono possibilità per la singola Società Medico Scientifica di modificare la situazione demografica dei (potenziali) membri, se non quelle legate alle attività di advocacy alta nel quadro delle influenze possibili sui Decisori. Nel caso della Società Italiana di Medicina di Laboratorio (SIMeL), l'esempio migliore è stato la stesura del "Patto per la modernizzazione e umanizzazione della Medicina di Laboratorio in Italia" [28], in occasione del Congresso Nazionale del 2004, inviato a tutti gli Assessorati delle Regioni italiane. In questo quadro generale di definizione e diffusione delle proprie "verità", si possono poi articolare alcune strategie specifiche per i singoli aspetti delle trasformazioni sociologiche societarie. Per l'aspetto dell'invecchiamento degli iscritti, i "vecchi" possono continuare a dare una mano, non nelle funzioni prettamente direttive ma nel supporto alle attività formative, scientifiche, amministrative, lasciando una testimonianza composta di vocazione (hanno scelto emotivamente il proprio campo d'impegno), fedeltà all'oggetto, tenacia, quasi di testardaggine nell'andare sempre nella stessa direzione, e serena continuità. Per superare alcune diffidenze all'iscrizione alla Società Scientifica da parte dei giovani professionisti, sono state suggerite linee di condotta per interessare i "trainees" alle Società, che vanno dagli sconti economici sulle prestazioni societarie, al maggiore coinvolgimento nelle attività scientifiche (incentivi e assistenza per poster e presentazioni), fino all'assunzione da parte di componenti della Società del ruolo di mentori per i giovani ricercatori e professionisti [22]. Per il tema di combinare adeguatamente gli interessi dei membri scientificamente attivi e di quelli non attivi, la soluzione più generale è nella strategia generale della Società (come sarà dettagliato più avanti) ma certamente un maggior coinvolgimento va cercato [21] come ad esempio, per SIMeL, con una ristrutturazione dei Gruppi di Studio (GdS) che consenta la convivenza fruttuosa delle diverse aspettative [29]. Comunque, ogni soluzione tecnica deve essere inserita in un clima interno caratterizzato dalla inclusività e dalla pari dignità di tutti i soci.

Sotto il profilo comunicativo si tratta di attrezzarsi per adeguare le forme tradizionali del networking (meeting e giornali) alle caratteristiche dell'era elettronica [12]. Per quanto riguarda i meeting, le soluzioni si incastrano, come si dirà, con il tema generale della formazione: da un lato, limitare gli incontri nazionali (anche se non dobbiamo dimenticare che nella ricerca di Bahr [22] "The number one reason for joining the society was to attend the annual meeting"), periferizzare i convegni e coinvolgere le organizzazioni periferiche nelle occasioni di incontro [30], puntare sull'apprendimento del saper fare che richiede il contatto interumano, combinare fasi di formazione di gruppo con richiami via web; dall'altro, riformare il concetto di Gruppo di Studio, facendo convivere il nucleo di gruppo di ricerca con una più ampia rete di condivisione di problemi che faccia sentire il coinvolgimento nella discussione e formazione degli statement [29]. Per quanto attiene i giornali, si tratta di combinare i vantaggi delle Società Scientifiche come editori (aderenza ai problemi reali della disciplina e indipendenza commerciale) con il superamento dei possibili svantaggi (ristrettezza del campo di interesse, limitata area di riferimento, resistenze al cambiamento) [31] tramite associazioni o accordi con editori importanti (per esempio con i Big Four: Elsevier, Springer, WileyBlackwell, Taylor \& Francis) e aprirsi ad una adeguata politica di "open access" e di utilizzo delle nuove forme di social network [32].

Per tutto questo, e per ciò che si dirà della formazione, è essenziale un Portale adeguato per funzionalità, potenzialità, manutenzione e contenuti costantemente aggiornati. Una ricerca [33] GIDIF-RBM (Gruppo Italiano Documentalisti dell'Industria Farmaceutica e degli Istituti di Ricerca Biomedica) del 2006 sulla qualità dei siti web di 228 Società Scientifiche Mediche e Veterinarie Italiane ha evidenziato una notevole diffusione e importanza dell'uso di siti web tendenzialmente informativi e una grande enfasi sulla progettazione grafica degli stessi, elaborati da studi di professionisti, ma scarsa attenzione alla qualità dei contenuti, in particolare carenze nell'aggiornamento dei testi (nel $70 \%$ dei casi!), scarsa attenzione alla accessibilità delle realizzazioni web (uso di frame, plugin, difficoltà di stampa), scarsa attenzione alla progettazione e performance bibliografico informativa. L'aspetto più grave, ha sottolineato la ricerca, è che il World Wide Web non è ancora percepito come uno strumento per favorire un'interazione diretta (uso di riferimenti "spersonalizzati”come info@indirizzo.it, mancanza di immagini del personale incaricato, mancanza di indicazioni di posta ordinaria e fax, impossibilità di utilizzo della clipboard di Windows, ecc.). Il Portale SIMeL oggi risponde a quasi tutti i criteri di valutazione usati dalla ricerca, solo in 
parte a quelli dell'aggiornamento continuo dei contenuti che deve essere compito condiviso dei soci (almeno di quelli attivi) della Società [34].

Per quanto attiene al ruolo nella formazione, le Società Medico Scientifiche sono meglio attrezzate per la rilevazione dei bisogni formativi, per la messa a punto di percorsi razionali e strutturati, per la verifica delle ricadute effettive della formazione, cioè esattamente i tre step cruciali di un' attività CME secondo la miglior letteratura (needs assessment, program design to met learner needs, outcome measurement) [35]. Il Documento 2007 su SIMeL e ECM [36] sostiene che Le Società Scientifiche possono garantire: 1) un'analisi dei bisogni capillare tra i membri interessati alla formazione, un contributo all'identificazione delle priorità, il rilevamento di temi emergenti; 2) la rilevazione della complessità e delle sfaccettature di un singolo argomento e la ricaduta nella sua applicazione su più categorie di Operatori, quando previste nell'articolazione societaria (medici, specialisti non medici, tecnici); 3) quindi, l'individuazione dei percorsi per disciplina; 4) l'attività di tutoraggio e tracce per la definizione di profili/percorsi formativi da proporre e/o adattare per i diversi Operatori presenti nelle Società Scientifiche; 5) una verifica nel tempo della penetranza dell'intervento e della sua ricaduta teorico-pratica; 6) investimenti specifici sulla formazione di formatori e/o tutor (formazione dei formatori); 7) la condivisione dell'onere formativo con altri Provider, con l'apporto di più punti di vista nella realizzazione dei progetti. La partecipazione alla Società Scientifica consente al singolo, utilizzando le risorse societarie, di determinare i suoi bisogni formativi in modo multidimensionale, attraverso la valutazione delle performance in confronto con le linee guida EBM che identificano gap e barriere ed evitano il potenziale "blind spot" del professionista, e chiudendo il cerchio di valutare l'effetto nella pratica attraverso metodi di feedback o di audit clinico [37]. Quanto all'esplicazione dei metodi formativi, la consapevolezza della "effectiveness" di CME è stata confermata da una ricerca sistematica [38] del 2007 effettuata dalla AHRQ (Agency of Healthcare Research and Quality) e ACCP (American College of Chest Physicians) che ha mostrato un'efficacia del $78 \%$ sulla conoscenza (68\% a lungo termine), del $71 \%$ sulle attitudini ( $68 \%$ a lungo termine), del $47 \%$ sulle abilità ( $70 \%$ a lungo termine), del $58 \%$ sulle performance (50\% a lungo termine) e del $42 \%$ sugli esiti clinici. Da tali dati sono state tratte le seguenti indicazioni e linee guida [39]. Nonostante l'eterogeneità degli studi e il basso grado delle evidenze, l'uso ripetuto di CME multimediale e multitecnica è efficace per migliorare ogni livello prestazionale. L'uso di un singolo strumento mediatico "live" ha un effetto di sviluppo professionale sia a breve che a lungo termine, mentre il singolo stru- mento cartaceo è inefficace. L'esposizione multipla moltiplica l'effetto e la durata dei cambiamenti professionali. Ciò ha importanti implicazioni per i provider di formazione: costruire i programmi intorno alle esigenze personali, ridisegnare i metodi integrandoli con strategie non educative, dedicarsi agli aspetti tecnologici e ambientali dell'apprendimento, avere l'autorevolezza della leadership nella professione [35]. Si tratta, per esempio, di variare l'offerta formativa in un mix di corsi residenziali e di FAD, puntare sugli incontri di apprendimento a piccolo gruppo del saper fare, combinare fasi di formazione di gruppo con richiami via web, promuovere una campagna di valutazione a distanza di tempo, inizialmente basata sulle procedure tradizionali o di self-assessment ma progressivamente sviluppata come verifica di performance e di outcome clinici.

Per quanto attiene gli aspetti scientifici, si è già detto dell'innovazione a livello di GdS e dell'opportunità di promuovere un mentoring dei colleghi più giovani. Per quanto riguarda SIMeL, sul piano dell'organizzazione orizzontale della Società, l'attenzione ai GdS rappresenta il principale trend dell'azione societaria: raddoppiati di numero, ristrutturati per dar modo a tutti di partecipare in forma attiva o passiva e di selezionare agevolmente i partecipanti desiderosi di transitare dal cerchio dell'informazione a quello della formazione, riconosciuti nel loro ruolo essenziale dalle riunioni annuali e dal loro coinvolgimento massiccio nella costruzione dei programmi scientifici dei Congressi a tema e del Congresso Nazionale. I GdS caratterizzano la multidisciplinarietà della Società, rappresentano concretamente il ruolo della SIMeL come forum di tutte le discipline che costituiscono la Medicina di Laboratorio: dall'ematologia alla microbiologia, dalla biochimica all'immunologia e così via. La possibilità di esistere dei GdS e di svolgere coerentemente il loro duplice ruolo di ricerca e formazione rappresenta la vita stessa di SIMeL. Una politica di sviluppo scientifico dei membri della Società dovrebbe svilupparsi anche collateralmente ai GdS. L'attenzione per la presentazione ed esposizione dei Poster in sede congressuale e la scelta di quelli meritevoli di essere trasformati in lavoro da pubblicare sulla Rivista della Medicina di Laboratorio sono stati i primi passi per incentivare e promuovere l'attività scientifica dei soci, in particolare i più giovani. Definizione di un preciso codice di condotta e mentoring formalizzato dovrebbero essere i passi successivi.

Sotto il profilo disciplinare, le Società Scientifiche devono adeguare ai tempi il corpus fondante della disciplina, essere normativi in quanto credibili (trasparenza e indipendenza), essere protagonisti dell'advocacy in senso alto.

Da questo punto di vista, SIMeL ha operato negli ulti- 
mi anni per la rifondazione dei fondamentali della disciplina, alla luce delle trasformazione mediche e sociali. Con riunioni e incontri, articoli scientifici, discorsi programmatici ma, soprattutto, con i tre primi Congressi Nazionali ha ribadito, riprendendoli ampiamente dalla lezione burliniana e aggiornandoli, i cardini della Medicina di Laboratorio, come risposta al quesito clinico. La risposta al quesito clinico, nella nuova visione, è il prodotto coordinato e condiviso di una equipe multidisciplinare e multiprofessionale tenuta insieme dalla propria mission e da una adeguata leadership, come interazione tra un trust di cervelli sollecitati dalla richiesta e dalla necessità di azione terapeutica del cervello del clinico, nella consapevolezza che nella organizzazione sanitaria di oggi il paziente è al centro di una rete assistenziale nella quale si dovrebbe spesso realizzare un equipe sinergica, piuttosto che l'azione del singolo medico. A questa visione che riprende, integrandole, le visioni di Lundberg, Büttner e Burlina, è stata aggiunta la sottolineatura, tutta moderna, della sicurezza del paziente, come altra faccia del concetto di qualità "globale", e l'attenzione per la conseguente organizzazione della Medicina di Laboratorio, scegliendo apertamente l'innovazione della Pathology Modernisation. Sul piano dell'organizzazione verticale della Società, questa visione ha maturato il concetto burliniano degli anni ottanta di forum multidisciplinare e multiprofessionale, coniugato con la difesa del medico di laboratorio, in una nuova visione del nuovo millennio di reale rappresentanza articolata e sinergica di tutte le professionalità fondanti la Medicina di Laboratorio, statutariamente riconosciute con pari dignità. Da ciò il concetto di "alleanza delle diversità", precetto valido sia all'interno che all'esterno della Società.

Sul piano dei rapporti con l'Industria, i tempi non consentono soluzioni radicali ma il conflitto di interessi deve essere affrontato. Un' assunzione di responsabilità è, ad esempio, il documento SIMeL 2007 sui Rapporti con l'Industria [40]. SIMeL ha definito come segue i punti di repere per la ridefinizione dei rapporti con l'Industria. $\mathrm{Si}$ ribadisce l'assoluta indipendenza di giudizio, di elaborazione concettuale e di valutazione di performance da parte di SIMeL relativamente ai contenuti tecnici, professionali, organizzativi ed etici della Medicina di Laboratorio. A tal fine SIMeL si impegna ad attuare sempre più estensivamente i contenuti dell'Appello 2003 del CIRB (Coordinamento per l'Integrità della Ricerca Biomedica) e il Documento sull'integrità della ricerca della FISM [4] con l'evidenza della dichiarazione dei conflitti di interesse nelle attività congressuali e degli strumenti informativi/formativi della Società (RIMeL e Portale). Si ribadisce l'impegno di SIMeL per la ridefinizione di una nuova ECM efficiente e trasparente, in cui unici Provider accreditati siano la Aziende del SSN e le
Società Scientifiche certificate. Enti commerciali/industriali non possono organizzare attività ECM né direttamente né indirettamente, ma possono dedicarsi ad attività promozionali e di training a loro più consone. Si ribadisce l'impegno di SIMeL a razionalizzare le attività congressuali e scientifiche. SIMeL da sempre ha eliminato la mostra strumentale dal proprio Congresso Nazionale ed è sempre stata molto attenta ai costi inerenti le attività congressuali, tenendo sotto stretto controllo la valorizzazioni delle opportunità offerte all'Industria. SIMeL, dal 1990, attua Corsi residenziali ispirati ai principi e alla prassi della formazione continua anglosassone, con rigorosa condotta sociale e intensivi apprendimenti sul campo. Deve essere chiaro che la razionalizzazione si attua sulla base della massimizzazione della qualità e che i depositari degli strumenti per giudicare la qualità e l'utilità degli eventi sono i professionisti e le Società Scientifiche, non l'Industria, che ovviamente giudica secondo il ritorno economico. Deve essere, inoltre, altrettanto chiaro che non è accettabile una razionalizzazione delle attività scientifiche delle Società Scientifiche per consentire una proliferazione di attività di Ditta, il cui contenuto scientifico è spesso di dubbio valore. Tali riflessioni e prese di posizione valgono nei confronti di tutti i soggetti commerciali, non solo dell'Industria del diagnostico e dei "medical devices", ma anche nei confronti dei provider commerciali di formazione, nei confronti delle case editrici e nei confronti delle aziende o agenzie di servizi con le quali la Società si rapporta. Per questo la Società deve essere dotata di una struttura indipendente (per SIMeL è la Segreteria) in grado di controllare la qualità e la correttezza dei servizi acquistati o condivisi. Chi si affida interamente e senza controllo ai fornitori fatica a mantenere indipendenza, trasparenza e credibilità.

Infine per quanto riguarda l'advocacy, vale la pena di ripetere che questa attività non va confusa con il sindacato e nemmeno con le associazioni professionali. Ciò che va difeso e diffuso è la vision della professione e della disciplina. È stato già citato il "Patto" [28] del 2004, ma altrettanto importanti sono stati i concetti espressi nelle "Linee di Indirizzo per la riorganizzazione dei Servizi di Medicina di Laboratorio nel Servizio Sanitario Nazionale" [41] e tutta una serie di contributi relativi, per citarne solo alcuni, all'essenza della Medicina di Laboratorio come risposta al quesito clinico, al ruolo e al fondamento dell'appropriatezza, alle basi etiche della Medicina di Laboratorio, al ruolo e alla roadmap dell'information technology nel Laboratorio, agli strumenti operativi della valutazione di efficienza ed efficacia, al ruolo delle concentrazioni e dei POCT nella medicina di laboratorio del nuovo millennio, alla interpretazione e gestione della Medicina Molecolare e delle omics fino al 
ruolo della Medicina di Laboratorio nella 4Ps Medicine [42]. L'insieme di questi e altri statement hanno fornito un solido background culturale agli adepti e una equilibrata guida per affrontare nella pratica le sfide scientifiche e organizzative, anche se non si è necessariamente trasformato in convinzione dei Decisori politici, stante le forze e i pregiudizi in campo. E tuttavia si può orgogliosamente sostenere che i comportamenti dei rappresentanti della Società sono stati coerenti con le linee guida e le posizioni teoriche sostenute. Non si sono assunti, cioè, comportamenti populisti e radicali con i componenti della disciplina e acquiescenza, se non collaborazione onerosa, con i Decisori. Forse questa dirittura morale e di proposta non è stata sufficientemente illustrata.

In conclusione, un futuro per le Società Medico Scientifiche esiste ed esso resta cruciale per la disciplina e i suoi adepti. Devono essere applicate alcune linee di azione per superare le ragioni pratiche dell'attuale crisi, che sono state sia pur sinteticamente indicate.

Ma vi sono alcune motivazioni profonde che devono essere rispettate perché il futuro si avveri:

1. Innanzitutto vi deve essere la coscienza del ruolo fondamentale della Società Scientifica nella rappresentazione della disciplina, con lo scopo di promuovere la ricerca, la formazione, la definizione di standard professionali e la verifica della qualità delle prestazioni [8]. Le Società Medico Scientifiche sono le depositarie e le custodi delle conoscenze e abilità e dei valori etici della disciplina, dell'educazione e della condotta scientifica degli adepti, della vision complessiva della specialità nell'ambito più generale della medicina e della società [23].

2. Le Società Scientifiche sono elementi di valore per l'intera società perché sono "differenti", sono in grado, cioè, di offrire un contributo intellettuale e materiale al benessere pubblico, indipendentemente da governi, comitati di ricerca, università, industria, e sono capaci di individuare gli obiettivi e i mezzi di massima efficacia e valore aggiunto [9]. In ultima analisi anche il potere di influenza sulle decisioni politiche dipende dalla credibilità che deriva dalla percezione dell'opinione pubblica che la Società lavora per l'interesse pubblico e non (solo) per interessi di gruppo più o meno evidenti [8].

3. Per le Società Scientifiche la chiave per continuare ad esistere è avere un ruolo per la scienza e per i propri soci. Solo se i leader e i membri delle Società Medico Scientifiche rimangono in continuo reciproco contatto per concordare linee di movimento, obiettivi coordinati e strategie ad ampio respiro, nel segno della più completa inclusività, i doveri e le prospettive della Società sono pienamente soddisfatti [21]. Le forzature e le ipocrisie, ancor peggio le dissimulazioni, come le esclusioni e le fazioni creano irreparabili fratture e impediscono di studiare insieme le soluzioni per $\mathrm{i}$ problemi pratici o strategici che via via si pongono.

4. Infine le Società devono essere governate con la trasparenza e l'applicazione dei principi e della prassi della democrazia partecipativa e della periferizzazione (in Italia oggi diremo federalismo) del coinvolgimento dei soci e delle attività scientifiche e formative [8]. Il governo degli "eletti" inaridisce presto la Società ad una conventicola di nessuna rilevanza. La accountability di persone e azioni deve essere la regola sempre seguita [43].

"Medicine is, in essence, a moral enterprise, and its professional associations should therefore be built on ethically sound foundation" [8].

\section{Conflitto di interesse Nessuno}

\section{Bibliografia}

1. Premuda L (1975) Storia della Medicina. CEDAM, Padova

2. Ornstein M (1928) Role of Scientific Societies in the Seventeenth Century. University of Chicago Press, Chicago

3. http://www.lib.uwaterloo.ca/society/. Accessed 16 October 2009

4. http://www.fism.it/cm/home.jhtml. Accessed 1 September 2011

5. http://www.alpsp.org/ngen_public/. Accessed 14 October 2009

6. Frankel MS (2000) Scientific Societies as sentinel of responsible research conduct. Proc Soc Exp Biol Med 224:216-219

7. Prados Castillejo JA (2010) Las sociedades cientificas y la gestion del conoscimiento, un paso màs allà del desarrollo profesional continuo. Aten Primaria. doi:10.1016/j.aprim.2009.09.018

8. Pellegrino ED, Relman AS (1999) Professional medical associations. JAMA 282:984-986

9. Fraser R (2000) Some threats and opportunities for learned societies in the new millennium. Microb Today 27:22-23

10. Schwartz MW, Hunter ML Jr, Boersma PD (2008) Scientific Societies in the 21st Century: a membership crisis. Conserv Biol 22:1087-1089.

11. McLendon WW (1999) Professional organizations, medical journals, and editors. A dynamic tension. Arch Pathol Lab Med 123:1141-1143

12. The future of Learned Societies. Columbia University 22 October 2009. www.youtube.com/watch?v=PrxXQuDGUhY. Accessed 1 September 2011

13. Young L, Everitt J (2004) Advocacy groups. University of British Columbia Press, Vancouver BC

14. Macrina FL (2007) Scientific Societies and promotion of the responsible conduct of research: codes, policies, and education. Acad Med 82:865-869

15. Levine FJ, Iutcovich JM (2003) Challenges in studying the effects of Scientific Societies on research integrity. Sci Eng Etichs 9:257268

16. Montori A, Onorato M (2008) Why there is a need of an Ethics Committee in Scientific Medical Societies. Dig Dis 26:32-35

17. Cappelletti P (2005). La risposta della Medicina di Laboratorio al quesito clinico. RIMeL-IJLaM 1(Suppl):15-23

18. Du Boulay C (2000) From CME to CPD: getting better at getting better? BMJ 320:393-394

19. Carbon C (2005) Continuing professional development and clinical governance: the role of scientific societies. Clin Microbiol Infect 11(Suppl 1):24-27 
20. Cappelletti P (2003) La Medicina Molecolare e la Medicina di Laboratorio. RIMeL-IJLM 4(S1):19-31

21. Hensen P, Stadler R, Luger TA (2009) The role and duties of scientific medical societies: The German Society of Dermatology from the members' perspective. JDDG. doi $10.1111 / \mathrm{j} .1610-$ 0387.2009.07210.x

22. Bahr JM (2008) Are scientific societies serving the needs of graduate and postdoctoral students? Reprod Fertil Dev 20:19-22

23. Relman AS (2007) Medical professionalism in a commercialized health care market. JAMA 298:2668-2670

24. ICMJE Uniform Disclosure Form for Potential Conflicts of Interest. http://www.icmje.org/. Accessed 17 October 2009

25. http://www.iom.edu/conflictofinterest. Accessed 17 October 2009

26. Rothman DJ, McDonald WJ, Berkowitz CD et al (2009) Professional Medical Associations and their relationship with Industry. A proposal for controlling conflict of interest. JAMA 301:1367-1372

27. Wofsy D (2005) Living in a different world. Arthritis Rheum 52:395-401

28. Cappelletti P (2005) Patto per la modernizzazione ed umanizzazione della Medicina di Laboratorio in Italia. RIMeL-IJLM 5:252-254

29. Regolamento dei GdS SIMeL. http://www.simel.it/it/documenti.php?testo $=\mathrm{LG}+05 \&$ cerca $=$ cerca. Accessed 5 September 2011

30. Carrol C, Noss RF, Hilty J, Trombulak SC (2009) Solving SCB's membership crisis by reinvigorating the Sections: response to Schwartz et al. Conserv Biol 23:5-6

31. Haynes JS (2010) Society Journal Publishing in the 21 st century. SPARC Japan Seminar, http://www.nii.ac.jp/sparc/en/event/2010/20101020en.html. Accessed 5 September 2011

32. Bizzaro N, Dorizzi RM (2011) La Rivista Italiana della Medicina di Laboratorio si rinnova: un nuovo editore e una nuova veste per continuare a crescere. RIMeL-IJLM 7:3-4

33. Ciappeloni R, Pistotti V, Bassi C et al (2006) La qualità dei siti web delle Società Scientifiche Mediche e Veterinarie italiane. XXIV Convegno GIDIF-RBM Portali come mezzo di comunicazione ed informazione: quale tipo, quali contenuti. Milano, 23 marzo 2006. http://veprints.unica.it/370/1/gidif.pdf. Accessed 2 August 2011

34. http://www.simel.it. Accessed 1 September 2011

35. Parker K, Parikh SV (2001) Applying Prochaska's model of change to needs assessment, program planning and outcome measurement. J Eval Clin Pract 7:365-371

36. Appunti per una nuova ECM. http://www.simel.it/it/documento.php/2064. Accessed 5 September 2011

37. Lowe MM, Aparicio A, Galbraith R et al (2009) The future of continuing medical education: effectiveness of continuing medical education: American College of Chest Physicians EvidenceBased Educational Guidelines. Chest 135(Suppl 3):69S-75S

38. Marinopoulos SS, Dorman T, Ratanawongsa N et al (2007) Effectiveness of continuing medical education. Evid Rep Technol Assess 149:1-69

39. Moores LK, Dellert E, Baumann MH, Rosen MJ (2009) Executive Summary. Effectiveness of continuing medical education: American College of Chest Physicians Evidence-based Educational Guidelines. Chest 135(Suppl 3):1S-4S

40. SIMeL e i rapporti con l'Industria. http://www.simel.it/it/documento.php/2064. Accessed 5 September 2011

41. http://www.agenas.it/innov_sperim_sviluppo/RiorganizMedicina_Laboratorio.pdf. Accessed 1 September 2011

42. Cappelletti P (2010) Medicina di Laboratorio. In: Plebani M, Galzigna L. Trattato Italiano di Medicina di Laboratorio. Volume I Biochimica Clinica e Generale. Piccin Nuova Libraria Spa, Padova,pp 9-104

43. Thorn S (2011) Governance of Learned Societies: Avoiding the Minefields and Enjoying Good Practice. Wiley-Blackwell Publishing news. http://blogs.wiley.com/publishingnews/2011/06/28/governance-of-learned-societies-avoiding-the-minefieldsand-enjoying-good-practice. Accessed 5 September 2011 